Case Report:

\title{
Traumatic Pseudoaneurysm of Peroneal Artery: A Case Report and Review of the Literature
}

\author{
Omid Mahmoudi Nasab ${ }^{\circledR}$, Mohsen Heidari®, Sam Haji Alilu Sami, Mohammad Mehdi Azizi ${ }^{\circledR}$, Mohammad Reza Kooshesh (D)
}

1. Bone and Joint Reconstruction Research Center, Shafa Orthopedic Hospital, Iran University of Medical Sciences, Tehran, Iran.

\begin{tabular}{|c|c|}
\hline $\begin{array}{l}\text { Usse your device to tscan } \\
\text { and read the article online }\end{array}$ & Crtation Mahmoudi Nasab O, Heidari M, Haji Alilu Sami S, Mehdi Azizi M, Kooshesh MR. Traumatic Pseudoaneurysm \\
\hline 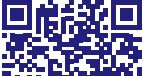 & $\begin{array}{l}\text { of Peroneal Artery: A Case Report and Review of the Literature. Journal of Research in Orthopedic Science. } 2019 ; 6(3): 13-16 . \\
\text { http://dx.doi.org/10.32598/JROSJ.6.3.13 }\end{array}$ \\
\hline 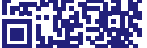 & deij http://dx.doi.org/10.32598/JROSJ.6.3.13 \\
\hline
\end{tabular}

\section{(i) (3)}

Article info:

Received: 27 Apr 2019

Revised: 29 Apr 2019

Accepted: 10 Jul 2019

Available Online: 01 Aug 2019

Keywords:

Pseudoaneurysm, Trauma,

Tumor like lesion

\begin{abstract}
A B S T RA C T
Introduction: Post-traumatic Pseudoaneurysm (PTP) is a rare condition. Because of its clinical course and presentation like a tumor lesion, its early diagnosis and appropriate treatment is necessary.
\end{abstract}

Case Presentation: In this study, we present a case of PTP in the left peroneal artery two years after trauma that presented with calf mass.

Conclousion: Magnetic resonance imaging and magnetic resonance angiography were used for the diagnosis, and the patient was successfully managed with endovascular stenting.

\section{Introduction}

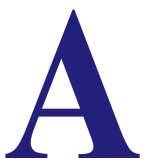

$\mathrm{n}$ aneurysm is a localized outward bulging occurred at a weak spot of the wall of a blood vessel. While a true aneurysm involves all three layers of the artery, a false aneurysm or pseudoaneurysm is a collection of blood confined between the two outer layers of an artery [1].

Pseudoaneurysm happens after the full-thickness tear or laceration of an artery, causing a pulsating hematoma connected to the lumen of the involved artery. The compression of neighboring neurovascular structures may cause neuropathy, venous obstruction, and thrombosis. The severity of symptoms depends on the size of the pseudoaneurysm [2]. Pain, swelling, and hematoma are the most common symptoms of the pseudoaneurysm. Several etiologies, including iatrogenic, traumatic, neoplastic, infective, vasculitic, and inflammatory have been known for pseudoaneurysm [3].

Post-traumatic Pseudoaneurysm (PTP) may occur several years after the trauma, making their diagnosis

* Corresponding Author:

Mohsen Heidari, MD.

Address: Bone and Joint Reconstruction Research Center, Shafa Orthopedic Hospital, Iran University of Medical Sciences, Tehran, Iran.

Phone: +98 (913) 1862040

E-mail: o.mayahi@gmail.com 
difficult. The clinical scenario may also resemble a softtissue tumor, thereby complicating the diagnosis [3-7].

Here, we describe a case of PTP involving the peroneal artery of the left leg of a 46-year-old male, who was presented with a soft-tissue mass two years after the initial trauma.

\section{Case Presentation}

A 46-year-old man was referred to our center with the complaints of pain at the posterior aspect of his left calf since two years ago, following a trauma caused by rebar. Since then, the back of his left calf was painful and swollen. The pain and swelling were exacerbated during the last three months.

The patient had no remarkable medical history. The results of laboratory tests, including cell blood count, erythrocyte sedimentation rate, and C-reactive protein, were all typical. In physical examinations, the skin over the mass was completely intact, and no ecchymosis or discoloration was present in this area. Neurologic examinations revealed the patient's normal strength and sensation. The swelling was not pulsatile and did not have any bruit. The posterior aspect of the left calf was tender in touch.

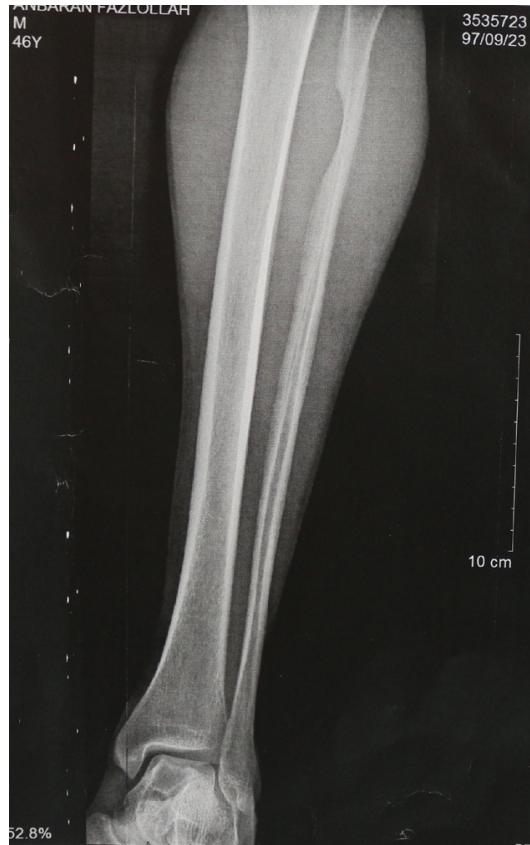

Orthopedic Science

Figure 1. Plain radiography shows compressive effect in the proximal fibula
Plain radiography of the left leg showed a soft tissue mass with a compressive effect in the proximal fibula (Figure 1).

An MRI was requested for the detailed evaluation of the lesion. The review of the MRI revealed a large (about $90 \times 68 \mathrm{~mm}$ ) round heterogeneous intensity mass in the proximal calf with turbulent flow artifact (Figure 2). An MR angiography was performed based on the MRI findings. Magnetic Resonance Angiography (MRA) revealed a heterogeneous mass about $90 \times 80 \mathrm{~mm}$ at the posterior aspect of the proximal leg that had a connection to peroneal artery representing a pseudoaneurysm, and the diagnosis of PTP of the peroneal artery was made (Figure 3).

The patient was successfully treated by endovascular stenting. While being discharged, the patient could walk without pain. Six months after the discharge, the patient was completely asymptomatic with no swelling or palpable peripheral pulses around the calf region.

\section{Discussion}

PTP is a lesion with a high rate of misdiagnosis, as they may appear a long time after the date of injury. Consequently, the differential diagnosis of these lesions, especially when resembling a soft-tissue tumor, is critical to prevent unnecessary invasive interventions. Angiography is an ideal tool for the diagnosis of PTP. However,

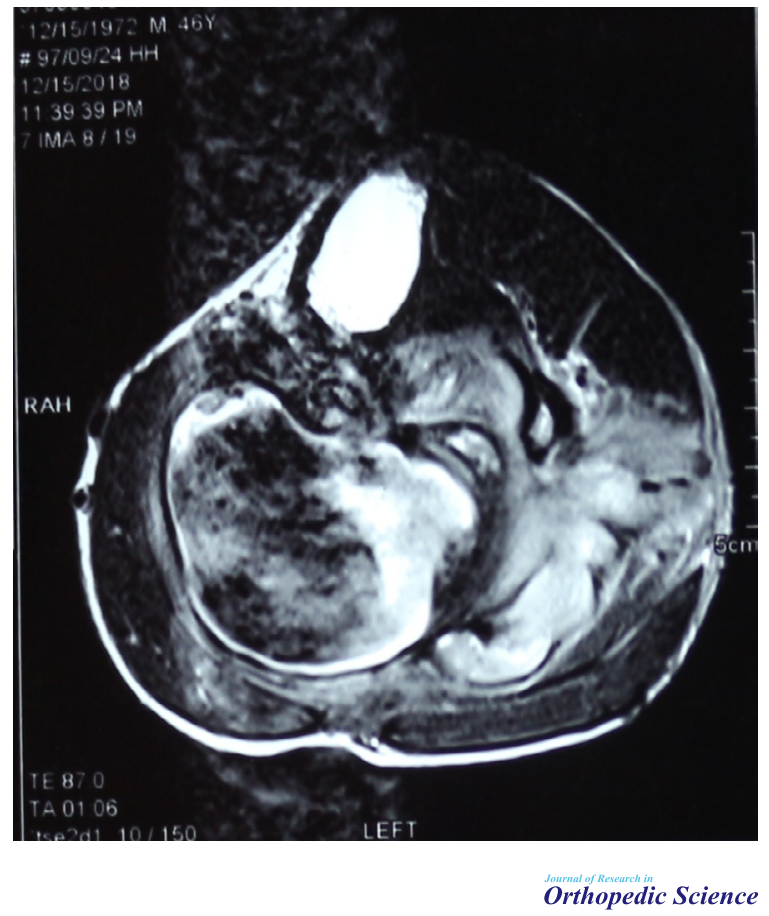

Figure 2. MRI axial cut shows a round heterogeneous intensity mass in proximal calf 


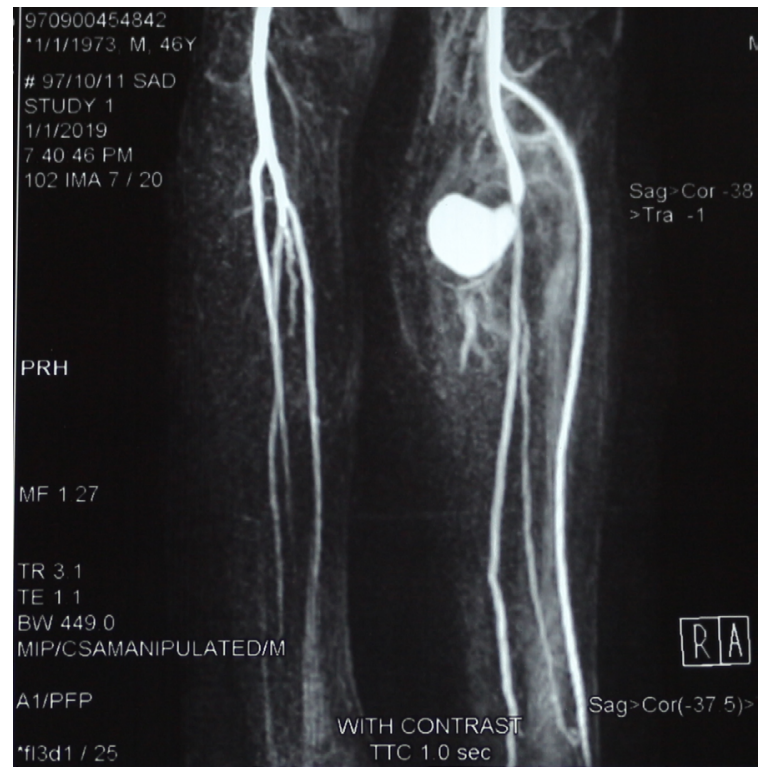

Orthopedic Science

Figure 3. MRA shows a heterogeneous mass about at posterior aspect of proximal leg that had a tract to proneal artery representing a pseudoaneurysm.

less invasive imaging studies such as MRI and MRA could be used in the diagnosis of PTP. In this respect, repetitive ghosting artifact in the phase-encoding direction that is related to the pulsation of the lesion are the critical features in MRI and a useful marker to suggest the presence of a high-flow vascular structure [7].

In this study, we presented a case of PTP of the peroneal artery in a 46-year-old man, who was referred to our center two years after the trauma. The diagnosis of the PTP was made by the observation of heterogeneous mass at the posterior aspect of the proximal leg that had a connection with the peroneal artery in MRA. The patient was treated by endovascular stenting. His symptoms were resolved afterward, and the 6-month follow-up of the patient was event-free. Our case revealed the importance of MRI and MRA as a noninvasive approach in the differential diagnosis of the PTP, mainly when the lesion resembles a soft-tissue tumor.

PTP of the posterior tibial artery has been rarely reported. Sadat et al. reported a case of peroneal pseudoaneurysm that occurred two weeks after thromboembolectomy with a Fogarty catheter. The diagnosis was made with a color Doppler ultrasound and complementary digital subtraction angiography. The lesion was successfully managed with an endovascular technique, including selective catheterization and coil embolization [8].
Ramdass reported a case of traumatic PTP of the peroneal artery in a 20-year-old man sustaining an injury to the right ankle while playing football. A pulsatile swelling was present over the affected region. The diagnosis was made by duplex scanning and MRI. A gadolinium contrast MRI of the right lower limb revealed a pseudoaneurysm at one of the terminal branches of the peroneal artery. The lesion was managed with the exploration of the pseudoaneurysm via a longitudinal incision in the lateral malleolus [9].

Albrecht et al. reported a case of PTP in the peroneal artery in the left lateral calf of a 35-year-old man 31 months after a gunshot wound. His MRI revealed a $7 \times 5 \times 4 \mathrm{~cm}$ pseudoaneurysm of the mid-left peroneal artery. The lesion was treated with the preoperative coil embolization of the feeding vessels and surgical decompression of the pseudoaneurysm via a $14 \mathrm{~cm}$ incision [10].

Shah et al. also reported a case of the PTP of the peroneal artery that occurred 12 weeks after open reduction and internal fixation of a trimalleolar fracture of the left ankle in a 33-year-old man. The diagnosis was confirmed by ultrasonography, and the anatomical site of the lesion was demonstrated by a digital subtraction angiogram. The pseudoaneurysm was successfully coiled [11].

Reviewing the literature reveals that MRI is less employed in the diagnosis of PTP. However, pseudoaneurysms can easily be detected with an MRI, using its unique features, including repetitive ghosting artifact in the phase-encoding direction $[7,12,13]$. In this study, we diagnosed the PTP of the peroneal artery, using MRI and MRA. Thus, MRI can be suggested as a reliable low-invasive approach in the diagnosis of PTP.

\section{Ethical Considerations}

\section{Compliance with ethical guidelines}

This study was approved by the Ethics Committee of Bone and Joint Reconstruction Research Center, Iran University of Medical Sciences, Tehran.

\section{Funding}

This research did not receive any specific grant from funding agencies in the public, commercial, or not-forprofit sectors.

\section{Authors' contributions}

All authors contributed in preparing this article. 


\section{Conflict of interest}

The authors declared no conflict of interest.

\section{References}

[1] Kalapatapu VR, Shelton KR, Ali AT, Moursi MM, Eidt JF. Pseudoaneurysm: A review. Curr Treat Options Cardiovasc Med. 2008; 10(2):173-83. [DOI:10.1007/s11936-0080019-8] [PMID]

[2] Raherinantenaina F, Rajaonanahary T, Ratsimba HR. Management of traumatic arterial pseudoaneurysms as a result of limb trauma. Formos J Surg. 2016; 49(3):89-100. [DOI:10.1016/j.fjs.2016.04.001]

[3] Albert S, Daniel S, Gouse M, Cherian VM. Case of pseudoaneurysm mimicking a soft tissue sarcoma: A diagnostic pitfall. Malays J Med Sci. 2015; 22(2):61-4. [PMID] [PMCID]

[4] Erler K, Ozdemir MT, Oguz E, Basbozkurt M. Does false aneurysm behave like a sarcoma? Distal femoral arterial false aneurysm simulated a malign mesenchymal tumor. Arch Orthop Trauma Surg. 2004; 124(1):60-3. [DOI:10.1007/s00402-003-0595-8] [PMID]

[5] Gantz E, Sweet M, Jakim I. False aneurysm mimicking an aggressive soft-tissue tumor. A case report. J Bone Joint Surg Am. 1988; 70(7):1090-2. [DOI:10.2106/00004623198870070-00020]

[6] Kim YJ, Baek WK, Kim JY, Park SW, Jeon YS, Lee KH, et al. Pseudoaneurysm of the popliteal artery mimicking tumorous condition. J Korean Surg Soc. 2011; 80(Suppl. 1):S71-S4. [DOI:10.4174/jkss.2011.80.Suppl1.S71] [PMID] [PMCID]

[7] Samim M, Mandell J, Smith S, Kapoor N, Czuczman G. Arterial pseudoaneurysms of the shoulder mimicking other entities: Utilization of pulsation artifact on musculoskeletal MR for accurate diagnosis in 2 cases. Skeletal Radiology. 2017; 46(8):1119-24. [DOI:10.1007/s00256-017-2622-9] [PMID]

[8] Sadat U, See T, Cousins C, Hayes P, Gaunt M. Peroneal artery pseudoaneurysm- a case report and literature review. BMC Surg. 2007; 7:4. [DOI:10.1186/1471-2482-7-4] [PMID] [PMCID]

[9] Ramdass MJ. Traumatic false aneurysm of the distal peroneal artery with associated anatomic anomaly: A case report. Cases J. 2009; 2:8605. [DOI:10.4076/1757-1626-28605] [PMID] [PMCID]

[10] Albrecht RJ, Parra JR. Traumatic peroneal artery pseudoaneurysm: Use of preoperative coil embolization. J Vasc Surg. 2004; 39(4):912. [DOI:10.1016/S0741-5214(03)00928-5]

[11] Shah A, Khan S, Adair W, Chatterji U. Peroneal artery pseudoaneurysm after surgical stabilisation of a Weber C Trimalleolar Ankle Fracture: A case report and review of literature. Trauma. 2014; 16(1):51-5 [DOI:10.1177/1460408613507687]
[12] Inamdar D, Alagappan M, Shyam L, Devadoss S, Devadoss A. Pseudoaneurysm of anterior tibial artery following tibial nailing: A case report. J Orthop Surg. 2005; 13(2):186-9. [DOI:10.1177/230949900501300216] [PMID]

[13] Melenhorst M, Van Der Mooren K, Van Nieuwenhuizen $\mathrm{R}$, Wüst A. Traumatic pseudoaneurysm following ankle trauma. BJR Case Rep. 2015; 1(3):20150132. [DOI:10.1259/ bjrcr.20150132] 